\section{Arthrose liegt in der Familie}

Bei starker familiärer Vorbelastung ist das Coxarthrosesrisiko schon in mittleren Lebensjahren deutlich erhöht, so eine prospektive britische Studie. Von den 123 Teilnehmer, deren Mutter oder Tante schon eine arthrosebedingterHüft-TEP hatte, wiesen $15 \%$ radiologische Zeichen einer Coxarthrose im mittleren Alter von 51 Jahren auf. Bei den unbelasteten Kontrollen waren nur $8 \%$ der Hüften auffällig. Klinische Symptome zeigten 15\% der erblich Belasteten und 5\% der Kontrollen. Osteoarthritis and Cartilage 2012 (online first)

\section{Erdbeerdiät gegen Lungenkrebs}

Jeden Tag ein paar Kilo Erdbeeren oder mehrere Liter Beerensaft können bei Raucher möglicherweise den Lungenkrebsrisiko senken. Zumindest rauchende Mäuse konnten in einer Studie dadurch Tumoren entgehen. Und zwar entwickelte dank der Schutzwirkung der Antioxidantien nicht mehr jede dritte, sondern nur noch jede siebte Raucher-Maus Lungenadenome. Zudem waren auch die zytogenetischen Schäden und die Leberdegeneration geringer ausgeprägt Int J Cancer 2012 (online first)

\section{Mütter mit Asthma? Trotzdem Stillen!}

Entgegen bisheriger Bedenken, weisen Kinder asthmakranker Mütter, die während vier Monaten oder länger gestillt worden sind, im Vergleich zu Kindern, die keine Muttermilch erhalten haben, bessere Lungenfunktionswerte im Schulalter auf. Eine Studie mit 1458 Schulkindern aus Großbritanien zeigt nämlich, dass sie im Schnitt ein umso größeres Atemzugvolumen hatten, je länger sie gestillt wurden. Am J Respir Crit Care Med 2012 (online first)

\title{
Riskante Schlafstörung
}

\section{Nachts 5 Mal wach? Dann droht Alzheimer!}

Wer schlecht schläft, weil er nachts häufig aufwacht, hat offenbar ein erhöhtes Alzheimerrisiko. Unklar ist allerdings, ob der schlechte Schlaf Alzheimer fördert oder ob der Untergang von Hirnzellen den Schlaf stört.

Vor allem häufige Unterbrechungen während des Schlafs gehen Hand in Hand mit der Bildung von Amyloid-

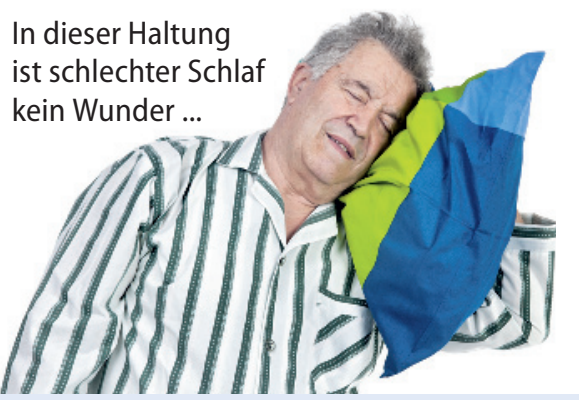

plaques im Gehirn, so eine aktuelle Studie, bei der bei 100 geistig gesunden Probanden im Alter von 45 bis 80 Jahren per PET und Liquortest nach Amyloid gesucht und über Wach- und Schlafzustand Buch geführt wurde.

Diejenigen Teilnehmer, die am häufigsten nachts aufwachten - zum Teil mehr als fünfmal pro Stunde - zählten überwiegend zu den $25 \%$, die bereits verdächtig viel Amyloid in ihrem Gehirn abgelagert hatten. Bei ihnen konnte man bereits eine präklinische Alzheimer-Erkrankung vermuten. Ob häufige Unterbrechungen ein erstes Symptom einer beginnenden Alzheimer-Demenz sind oder ob Schlafstörungen die AlzheimerPathogenese beschleunigen ist allerdings noch unklar. (mut) www.aan.com/globals/axon/assets/9286.pdf

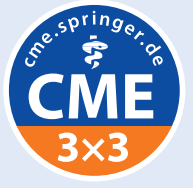

\section{CME-Punkte kostenlos in jeder Ausgabe}

\section{CME hilft Ihnen, Ihr Punktekonto zu füllen!}

Mit unseren drei zertifizierten Fortbildungen können Sie pro Heft auf CME.springer.de/CME kostenlos 9 Punkte sammeln. Und so geht's:

\section{Kostenlos teilnehmen in vier Schritten}

\section{Auswählen}

Wählen Sie online auf CME.springer. de/CME den Link zum aktuellen Heft aus. Entscheiden Sie nun, welche Fortbildungseinheiten Sie zum Erwerb von CME-Punkten nutzen wollen. Die Teilnahme an den drei Einheiten der aktuellen Ausgabe ist kostenlos.

\section{Registrieren/Anmelden}

Sind Sie bereits bei CME.springer.de registriert? Dann genügt zur Anmeldung die Angabe Ihrer persönlichen Zugangsdaten. Falls Sie zum ersten Mal teilnehmen, bitten wir Sie, sich einmalig zu registrieren. Wir senden Ihnen dann per E-Mail Ihre persönlichen Zugangsdaten zu. Bitte benutzen Sie diese für alle weiteren Teilnahmen zur Anmeldung (Login).

\section{Teilnehmen}

Sie können die elektronische Version der Fortbildung online lesen, ausdrucken oder direkt die 10 CME-Fragen beantworten. Die kostenlose Teilnahme ist 1 Monat nach Erscheinen der Zeitschrift möglich.

\section{CME-Punkte sammeln} Nach erfolgreicher Beantwortung von 7 der 10 CME-Fragen senden wir Ihnen Ihre Teilnahmebestätigung samt Punkten umgehend per E-Mail zu. Diese können Sie bei Ihrer zuständigen Landesärztekammer einreichen. 